\title{
THE SUPPORT VECTOR MACHINE PARAMETER OPTIMIZATION METHOD BASED ON ADAPTIVE ELITIST DIFFERENTIAL EVOLUTION ALGORITHM AND ITS APPLICATION TO ROLLER BEARING FAULT DIAGNOSIS
}

\author{
HUNGLINH AO ${ }^{1, *}$, THANHHANG NGUYEN ${ }^{1}$, V.HO HUU ${ }^{2}$, TRANGTHAO NGUYEN ${ }^{2}$ \\ ${ }^{1}$ Faculty of Mechanical Engineering, Industrial University of Ho Chi Minh City, \\ ${ }^{2}$ Institute for Computational Science, Ton Duc Thang University. \\ aohunglinh@iuh.edu.vn
}

\begin{abstract}
SVM parameters have serious effects on the accuracy rate of classification result. Tuning SVM parameters is always a challenge for scientists. In this paper, a SVM parameter optimization method based on Adaptive Elitist Differential Evolution (AeDE-SVM) is proposed. Furthermore, AeDE-SVM is applied to diagnose roller bearing fault by using complementary ensemble empirical mode decomposition (CEEMD) and singular value decomposition (SVD) techniques. First, original acceleration vibration signals are decomposed into Intrinsic Mode Function (IMFs) by using CEEMD method. Second, initial feature matrices are extracted from (IMFs) by singular value decomposition (SVD) techniques to obtain single values. Third, these values serve as input vector for AeDE-SVM classifier. The results show that the combination of AeDE-SVM classifiers and the CEEMD-SVD method obtains higher classification accuracy and lower cost time compared to other methods. In this paper, the roller bearing vibration signals were used to evaluate the proposed method. The experimental results showed that the superior performance compared to other SVM parameter optimization techniques and successfully recognized different fault types of roller bearing during its operation.
\end{abstract}

Keywords. AeDE, CEEMD, Roller Bearing Fault, SVD, SVM.

\section{INTRODUCTION}

Support vector machine (SVM), one of the powerful techniques for regression and classification in machine learning, has been widely used in recent decades. SVM is the binary classification algorithm implemented based on the theory of risk minimization to find an optimal separation hyperplane in a multi-dimensional space. It has showed the advantages in high dimensional classification problems compared to other methods, such as logistic regression[1], k-nearest neighbors[2] by the high generalization capability and sparse representation ability. However, in various applications, SVM always requires different suitable parameters in order to achieve the best classification rate. In another word, the quality of SVM classification is significantly affected by the selection method of its operating parameters, including ordinary SVM parameters and kernel function parameters. Unfortunately, there is currently no common method for selecting the SVM parameters which can ensure SVM performance in various problems. Therefore, it is essential to develop a dynamic method for optimizing the SVM parameters in various applications.

Tuning SVM parameters is one of the major challenges for scientists in several decades. Several approaches have been proposed to provide a general SVM parameter optimization method, but still got some drawbacks. The standard method grid-search, although providing a good performance, requires complex computation and time consuming. Gradient-based approaches can also be used, such as simulated annealing, but tends to be trapped in complicated scoring for assessing the performance of the parameters [3-5]. Evolutionary algorithms, a class of iterative, randomized, global optimization techniques[6],or the heuristic algorithms, such as the genetic algorithm (GA), the particle swarm optimization (PSO), and the ant colony optimization (ACO) were also used to optimize SVM parameters[7, 8]. However, they could be easily stranded in local optimization areas and required high computation cost.

This research aims to provide a proper method that can generally tune and optimize SVM parameters. Among several techniques have been developed and successfully applied for a variety of structural optimization issues, such as Sequential Linear Programming (SLP)[9], sequential quadratic programming (SQP)[10], optimality criterion (OC)[11], and coercive methods; the Adaptive Elitist Differential Evolution (AeDE) algorithm - the improved version of differential evolution (DE) introduced by Storn and Price in 
1977 - is one of the most appropriate methods to solve existing issues[12, 13]. Developed to solve the problems of nonlinear constraints and discrete variables, the DE related methods have been proven as an effective method in addressing many technical problems, especially AeDE with the efficient and effective performance in handling discrete variables[14]. AeDE was improved from the DE in mutation and selection phase to enhance selection capability and convergence rate so that the computational cost and time consumption would be significantly reduced. Therefore, in this method, the AeDE was integrated in SVM training process to simultaneously obtain the optimized SVM parameters.

In order to demonstrate the superior performance of AeDE-SVM, roller bearing vibration signals were used for detecting four different fault types. First, the collected acceleration vibration signals from the roller bearing were fed to feature extraction subsystem which was built based on the Complementary Ensemble Empirical Mode Decomposition (CEEMD) method to decompose into Intrinsic Mode Functions (IMFs). Then, by the singular value decomposition (SVD) techniques, single value vectors were obtained from initial feature matrices extracted from the IMFs. Finally, AeDE-SVM classifier, with input from those single values, was used to detect fault types. The results show that the combination of AeDE-SVM classifiers and the CEEMD-SVD method obtains higher classification accuracy and lower cost time compared to other methods.

\section{ADAPTIVE ELITIST DIFFERENTIAL EVOLUTION}

The Adaptive Elitist Differential Evolution is an improved version of the differential evolution algorithm with two innovations in mutation phase and selection phase to enhance selection and optimization capability for discrete variables. In evolutionary computation, the DE is a method of optimization by repeatedly improving a quality-related candidate solution. It is, in fact, an iterative process, including initialization, mutation, crossover, and selection processes, to find the global search solution for general optimization problems. However, DE parameters, for example mutation factor F, crossover control parameter CR and trial vector generation strategies, have a significant impact on its performance. To overcome the common limitations of optimization algorithms, such as the use of a huge amount of resources as well as high computational cost, the AeDE was proposed with two improvements. The first one - adaptive technique based on the difference of the objective function between the best individual and the whole population in the previous generation - was applied in the mutation phase to improve the search capability. The second one - the optimum technique for selecting the best individuals for the next generation - was applied in the selection phase to enhance the search capability and to increase the convergence rate.

The new adaptive mutation scheme of the DE used two mutation operators. The first one was the "rand/1" which aims to ensure diversity of the population and prevents the individual from being trapped in an optimal local location. The second one was the "current-to-best/1" which accelerates convergence speed of the population by leading the population to the best individuals. On the other hand, the new selection mechanism always searched and stored the best individuals of the whole population as the reference for next generation orientation which fastens the convergence. The children population $\mathrm{C}$ containing of trial vectors was combined with the parent population $\mathrm{P}$ of target vectors to create a combined population $\mathrm{Q}$. Then, from the $\mathrm{Q}$, best individuals NP were selected to construct the population for the next generation. The elitist selection operator was shown in Algorithm 1.

Table 1: Algorithm 1: Elitist Selection Operator

1: Input: Children population $\boldsymbol{C}$ and parent population $\boldsymbol{P}$

2: Assign $\boldsymbol{Q}=\boldsymbol{C} \cup \boldsymbol{P}$

3: Select $\boldsymbol{N P}$ best individuals from $\boldsymbol{Q}$ and assign to $\boldsymbol{P}$

4: Output: $\boldsymbol{P}$

Table 2: Algorithm 2: The Adaptive Elitist Differential Evolution (AeDE) Algorithm

1: Initialize the population

2: Evaluate the fitness for each individual in the population

3: while delta $>$ tolerance or MaxIter is not reached do

// Definition of searching criteria

4: for $\mathrm{i}=1$ to NP do

5: $\mathrm{F}=\operatorname{rand}[0.4,1]$

// Find the best individuals

// Generate the initial mutation factor 


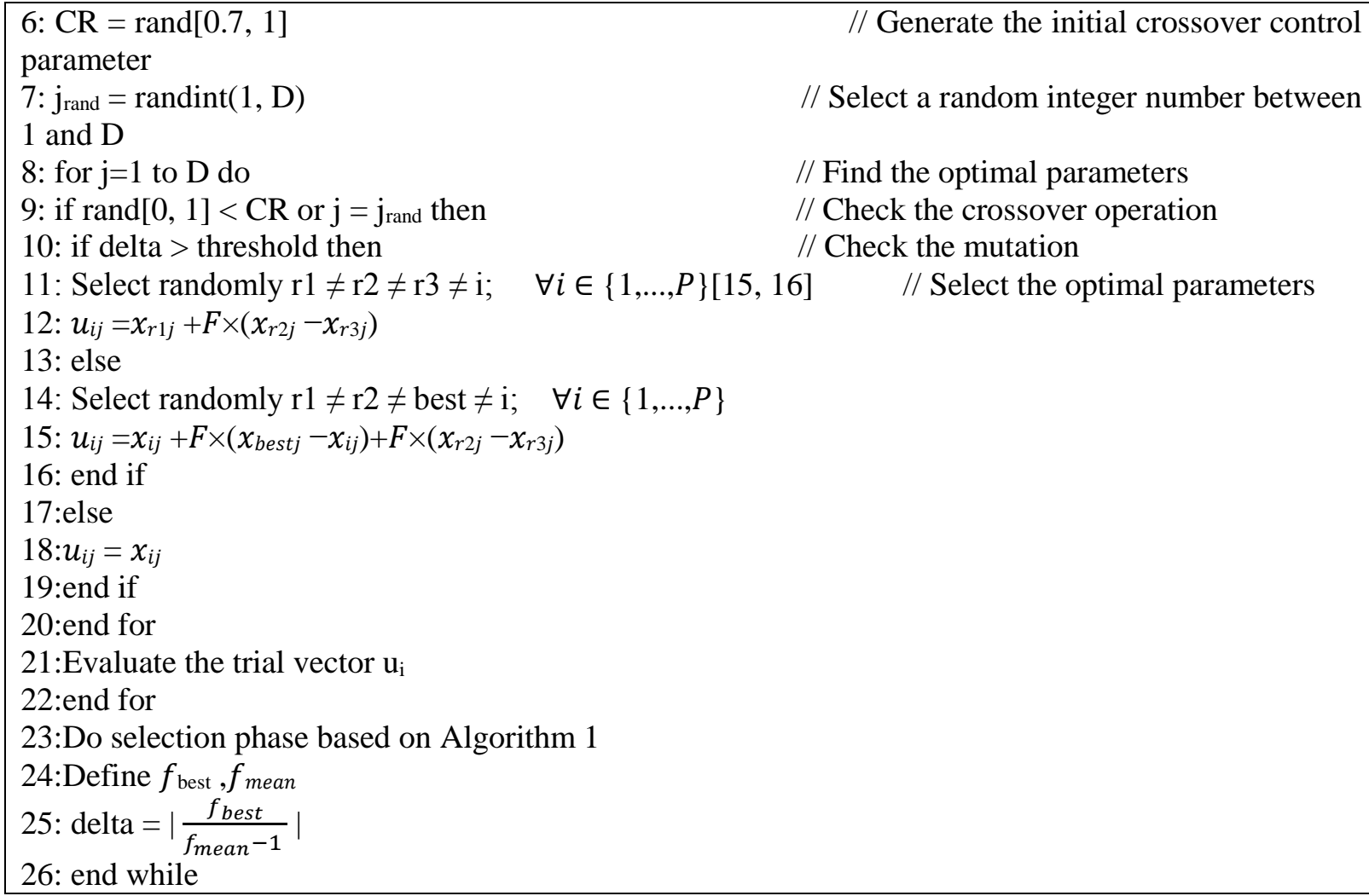

where tolerance is the allowed error; MaxIter is the maximum number of iterations; and $\operatorname{randint}(1, \mathrm{D})$ is a function that returns a uniformly distributed random integer between 1 and $\mathrm{D}[12]$.

\section{AEDE BASED SVM PARAMETER OPTIMIZATION}

\subsection{Support Vector Machine}

Since firstly introduced by V.N.Vapnik, the SVM has become one of the most popular types of machine learning based on the concepts in statistics and computer science. It is a supervised learning method with associated learning algorithms used for classification and regression analysis. By the basic idea of separating the given problem domain into two opposite signed half-spaces (positive and negative spaces) by only a few indicators, called support vectors, SVM shows their superior advantages in noisy data and outstanding performance in sparse representative[1,2, 17]. However, choosing the SVM parameters which have a significant impact on the accuracy of classification result, is never an easy task.

Standard binary SVMs accept input vectors and classify them into two different classes by a sign function. By using the mapping function $\varphi$, SVM actually maps training patterns from input space into a higherdimensional feature space to increase the class separation. Assume that there was a training sample set $G$ $=\left\{\left(x_{i}, y_{i}\right) ; i=1,2, \ldots, l\right\}$, where each sample $\mathrm{x}_{\mathrm{i}} \in \mathrm{R}^{\mathrm{d}}$ belonged to a class by $\mathrm{y} \in\{+1 ;-1\}$; and the training data were not well-separated in input feature space, then the objective function could be as the following:

$$
\begin{gathered}
\text { Minimize } \phi(\omega)=\frac{1}{2}\langle\omega \mid \omega\rangle+C \sum_{i=1}^{l} \xi_{i} \\
\text { subject to } y_{i}\left(\left\langle\omega . \phi\left(x_{i}\right)\right\rangle+b\right) \geq 1-\xi_{i}, \quad \xi_{i} \geq 0, i=\{1,2, \ldots, l\}
\end{gathered}
$$

where $\omega$ was the normal vector of the separating hyperplane, $C$ was the penalty coefficient parameter, $b$ was the bias, $\xi_{\mathrm{i}}$ were nonnegative slack variables, and $\varphi(\mathrm{x})$ was the mapping function[7].

By applying a non-negative Lagrange multipliers $\alpha_{i} \geq 0$, the optimization problem could be rewritten as follows: 


$$
\begin{gathered}
\text { Maximize } L(\omega, b, \alpha)=\sum_{i=1}^{l} \alpha_{i}-\frac{1}{2} \sum_{i, j=1}^{l} \alpha_{i} \alpha_{j} y_{i} y_{j} K\left(x_{i}, x_{j}\right) \\
\text { subject to } 0 \leq \alpha_{i} \leq C, \sum_{i=1}^{l} \alpha_{i} y_{i}=0
\end{gathered}
$$

The decision function can be obtained as:

$$
f(x)=\operatorname{sgn}\left[\sum_{i=1}^{l} \alpha_{i} y_{i} K\left(x_{i} \cdot x\right)+b\right]
$$

In the above equation, the most common kernel function, the radial basis (RBF) kernel function, was used to transform the initial problem domain to Gaussian domain, as shown in the following equation

$$
K\left(x, x_{i}\right)=\exp \left(\frac{-\left\|x-x_{i}\right\|^{2}}{2 \sigma^{2}}\right)
$$

where $\sigma$ is the kernel parameter.

\subsection{AeDE based SVM Parameter Optimization}

As mentioning in previous section, it is widely known that the SVM parameters strongly affect to the performance. However, there is currently no general - dynamic technique to choose these parameters. Many researchers used brute-force or random trial-error optimization technique, which required long time processing and huge computational cost. In this paper, we introduced AeDE as a method to optimize SVM parameters.

Particularly in the RBF kernel SVM approach, the penalty factor $\mathrm{C}$ and the kernel parameter $\sigma$ in the Gaussian kernel function could be considered as the optimization variables while testing error was the optimization problem fitness measurement, given as follows:

where

$$
\text { fitness }(C, \sigma)=\text { Test_Error }_{S V M}(C, \sigma)
$$

$$
\text { Test_Error }_{S V M}=\frac{\text { Number of incorrect classification in test samples }}{\text { Total number of samples in test set }}
$$

In general, the AeDE algorithm was integrated to SVM training procedure to obtain the optimal parameters for maximizing the classification accuracy and generalization capability of the SVMs. Initially, each individual in the first generation is randomly obtained. The SVM algorithm normally calculated the corresponding output weights matrix for each individual. Then, AeDE can be applied to find the fitness measurement for each individual in the population. This process was repeated until the stopping condition was reached. When the evolution is finished, the optimal parameters of the SVM were ready to perform the classification[7]. The procedure of AeDE-SVM algorithm is shown as follow:

Table 3: Algorithm 3: AeDE-SVM optimization algorithm

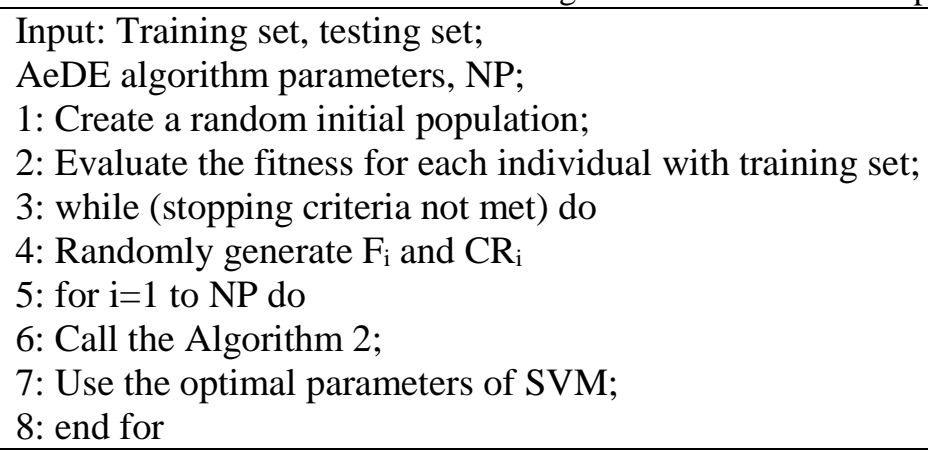


9: end while

10: Evaluate the optimized model by testing set;

Output:

Classification result

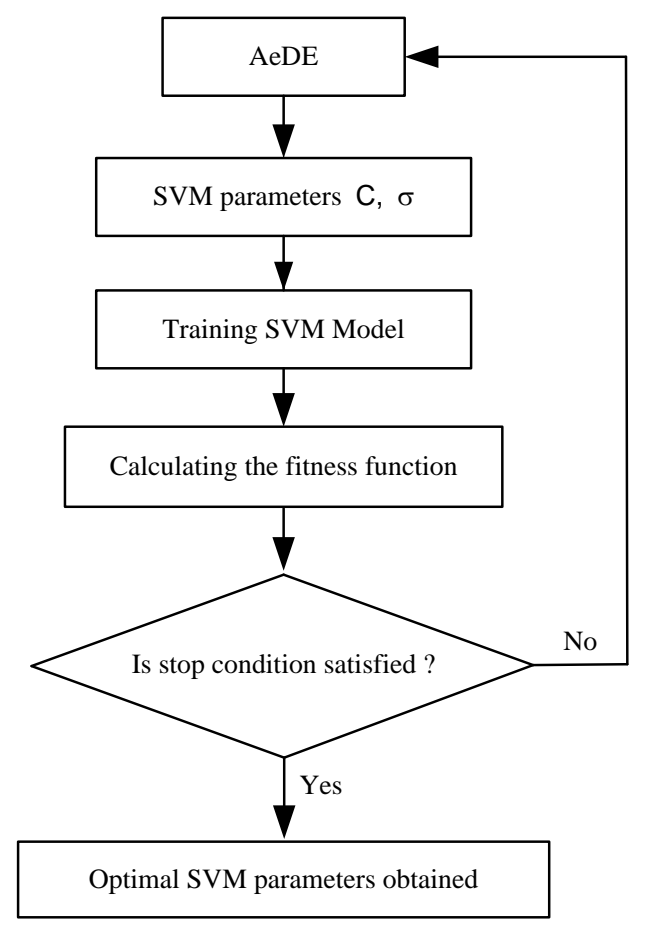

Figure 1: The parameter optimization flowchart of SVM based on AeDE.

\section{APPLICATION OF AEDE-SVM IN ROLLER FAULT DIAGNOSIS ANALYSIS}

\subsection{System Overview}

Machine learning as well as prediction techniques have shown their advantages in machinery fault detection recently to avoid and reduce the risks and costs of unexpected machine damages. In this paper, the AeDESVM was used to detect the roller fault, one of the most popular components in industrial applications.

The collected roller bearing acceleration vibration signals were decomposed into a specific number of IMFs by using CEEMD method. Then, the SVD technique was used to perform a dimensionality reduction and to provide the set of single value vectors, later on used as the input vectors of AeDE-SVM classifier.

\subsection{Complementary Ensemble Empirical Mode Decomposition (CEEMD)}

Complementary ensemble empirical mode decomposition is an improved algorithm of empirical mode decomposition. The EMD related approaches, mainly developed for nonlinear and nonstationary data, are empirical, intuitive, direct and self-adaptive comparing to other traditional decomposition techniques, such as Fourier transform or Wavelet transform. Basically, the EMD can decompose any time series signal into a finite number of IMFs by an iterative sifting process. Beginning with an assumption that any time series signal consists of different modes of oscillations concomitant simultaneously due to intrinsic complexity hidden in the data[18]. Those concomitant oscillatory functions, also called IMFs, can be extracted by EMD, shown in the equation.

$$
x(t)=\sum_{i=1}^{n} i m f_{i}(t)+r_{n}(t)
$$

where $x(t)$ is the vibration signal, $\operatorname{imf}_{i}(t)$ is the $i^{\text {th }}$ IMF component in different frequency bands ranging from high to low, and $r_{n}(t)$ is the $\mathrm{n}^{\text {th }}$ residue of the decomposition process, which is the mean trend of $x(t)$, 
and $n$ is the number of decomposition steps as well as the total number of IMFs. In order to be successfully extracted, IMFs should satisfy two mandatory requirements. Firstly, the number of extrema (including maxima and minima) and the number of zero-crossing should be equal or differ at most by one. Secondly, the average of the envelopes composed of the maxima and minima should be zero. This decomposition process was repeated until the last data series $r(t)$ could not be decomposed, indicating the end of the sifting process[19]. Despite the robustness of EMD, it was usually suffered by the mode mixing problem, which is defined as either a single IMF consisting of widely disparate scales or signal residing in different IMF components[20].

To overcome the problem of mode mixing, the ensemble empirical mode decomposition (EEMD) was proposed, where Gaussian white noises with finite amplitude are added to the original signal during the entire decomposition process. Due to the uniform distribution statistical characteristics of the white noise, the signal with white noise becomes continuous in different time scales, and no missing scales are present. As a result, mode mixing is effectively eliminated by the EEMD process [18]. It should be noted that, during the EEMD process, each individual trial may produce noisy results, but the effect of the added noise can be suppressed by large number of ensemble mean computations, in another word, too time consuming to implement.

An improved algorithm, CEEMD, is suggested to improve the computation efficiency. In this algorithm, the residue of the added white noises can be extracted from the mixtures of data and white noises via pairs of complementary ensemble IMFs with positive and negative added white noises. Although this new approach yields IMF with a similar RMS noise to EEMD, it eliminates residue noise in the IMFs and overcomes the problem of mode mixing with much more efficiency [14]. The procedure on implementing CEEMD is defined as the following:

- $\quad x_{1}$ and $x_{2}$ are constructed by adding a pair of opposite phase Gaussian white noises $x_{n}$ with the same amplitude.

$$
\left\{\begin{array}{l}
x_{1}=x+x_{n} \\
x_{2}=x-x_{n}
\end{array}\right.
$$

- (b) $x_{1}$ and $x_{2}$ are decomposed by EMD only a few times, and $\mathrm{IMF}_{x 1}$ and $\mathrm{IMF}_{x 2}$ are ensemble means of the corresponding IMF generated from each trial;

- (c) the average of corresponding component in $\mathrm{IMF}_{x 1}$ and $\mathrm{IMF}_{x 2}$ is calculated as the CEEMD decomposition results $[15,16]$; that is,

$$
I M F=\frac{\left(I M F_{x_{1}}+I M F_{x_{2}}\right)}{2}
$$

\subsection{Single Value Decomposition (SVD)}

The SVD technique is a matrix decomposed to generate singular values, singular vectors, and their relation to SVD.

Assuming there was a matrix $\Sigma$, which had $\mathrm{M} \times \mathrm{N}$ dimension, and was indicated as:

$$
\Sigma=E \Delta V^{T}
$$

where $E=\left[e_{1}, e_{2}, e_{3}, \ldots, e_{n}\right] \in R^{N \times N}, E^{T} E=I, V=\left[v_{1}, v_{2}, v_{3}, \ldots, v_{n}\right] \in R^{M \times M}, V^{T} V=I, \Delta R^{N \times M}$, $\Delta=\left[\operatorname{diag}\left\{\sigma_{1}, \ldots, \sigma_{p}\right\}: 0\right], p=\min (N, M)$, and $\sigma_{1} \geq \sigma_{2} \geq \ldots \geq \sigma_{p} \geq 0$. The $\mathrm{i}^{\text {th }}$ left and right singular vectors of matrix $\Sigma$ were vectors $e_{i}$ and $\mathrm{v}_{\mathrm{i}}$, respectively. The values of $\sigma_{\mathrm{i}}$ were the singular values of the matrix $\Sigma[7]$.

In this research, CEEMD method is recalled to decompose the roller bearing signals into several Intrinsic Mode Functions (IMFs), as shown in. All of the IMFs obtained from CEEMD method then were divided into two initial feature vector matrices $\mathrm{X}$ and $\mathrm{Y}$ 


$$
X=\left[\begin{array}{c}
I M F_{1} \\
I M F_{2} \\
\vdots \\
I M F_{J}
\end{array}\right], \quad Y=\left[\begin{array}{c}
I M F_{J+1} \\
I M F_{J+2} \\
\vdots \\
I M F_{n}
\end{array}\right]
$$

where $J=n / 2$ (when $\mathrm{n}$ is an even number) and $J=(n+1) / 2$ (when $\mathrm{n}$ is an odd number). Here, from the initial feature vector matrices $\mathrm{X}$ and $\mathrm{Y}$, the characteristic of the roller bearing vibration signal $\mathrm{x}(\mathrm{t})$ could be extracted. Additionally, fault feature vectors could be found as the singular values that reflect the nature characteristics of the vector matrices $\mathrm{X}$ and $\mathrm{Y}$ and the roller bearing vibration signal. After obtain fault feature vectors, the AeDE-SVM classifier could be used to identify the working condition and fault pattern of roller bearing $[7,15,16]$.

Figure 2 showed the flow chart of the roller bearing fault diagnosis method based on CEEMD-SVD and AeDE-SVM.

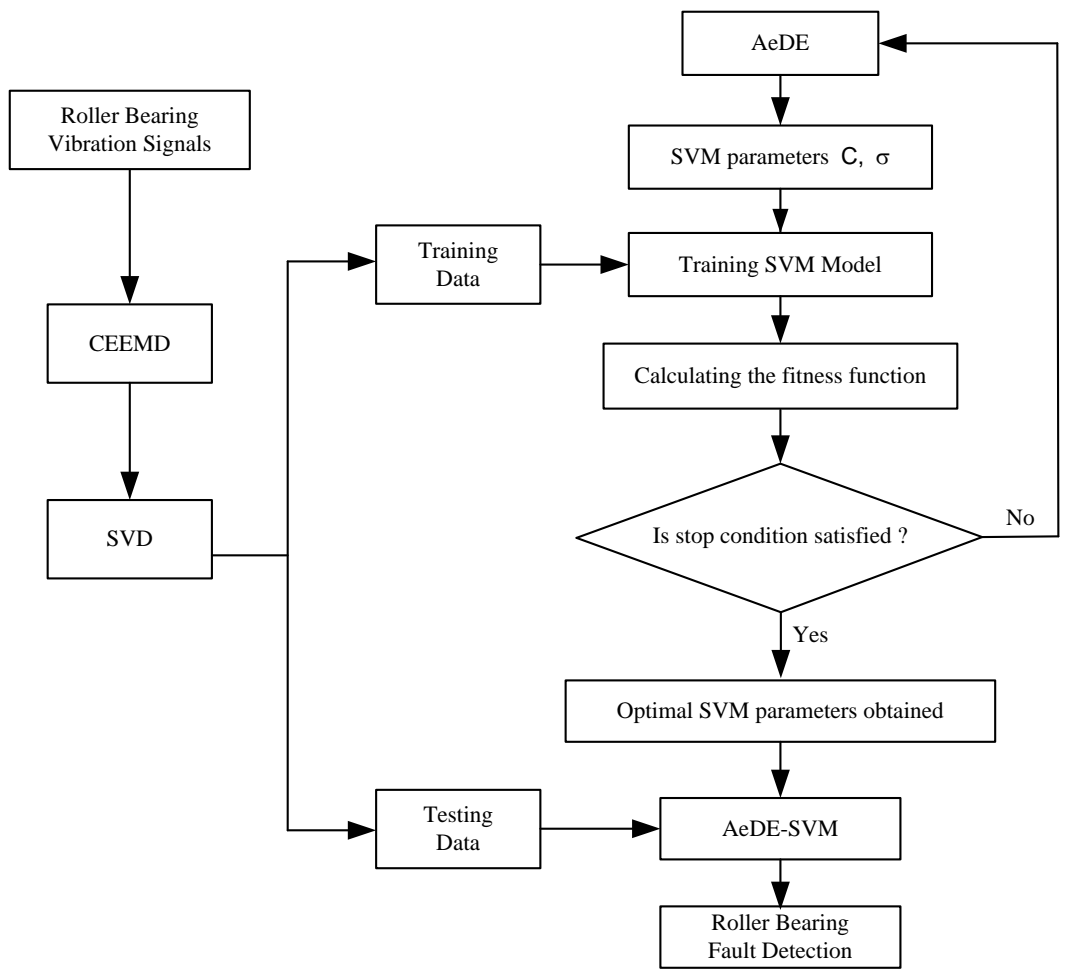

Figure 2: Roller Bearing Fault Detection Method Based on CEEMD-SVM and AeDE-SVM

\section{EXPERIMENTS AND RESULTS}

\subsection{Dataset}

A dataset from the Case Western Reserve University - Bearing Data Center website (CWRUBDCW), under Professor K.A.Loparo's permission, was used in this project to demonstrate the proposed method performance. The testing model contained a 2 HP Reliance Electric Motor, a torque transducer/encoder, a dynamometer and electronic controllers. An analog to digital converter was also used at $485063 \mathrm{~Hz}$ sampling rate, while the motor speed was fixed at $1772 \mathrm{rpm}$. Besides, a deep groove ball bearing (from SKF) and drive end bearings, 6205-2RS JEM type, were also used in this test. The test bearing of electrodischarge machining with fault diameter of 0.007 inches was selected. Four different roller bearing conditions were applied in this test in order to provide 80 various vibration signals in each different conditions. Finally, 56 groups were randomly selected for training while the remaining was reserved for testing. 


\subsection{Experiments and Results}

In fact, a binary AeDE-SVM, as an improved SVM, can only solve 2-class problems, thus, at least 3 AeDESVM must be used to identify 4 different operating conditions of roller bearing. In this experiment, a combined classifier, including three binary AeDE-SVMs, has been used to subsequently separate four different bearing operating conditions. The AeDE-SVM1 only identified either inner race fault or not. The non-fault patterns were then fed to AeDE-SVM2 for recognition of outer race fault. As the same manner, the AeDE-SVM3 received the non-fault patterns and classify them as normal condition or ball fault cases. The entire classification model was clearly showed in the Figure 3.

Besides, some other SVM parameter optimization methods, such as genetic algorithm (GA-SVM) and particle swarm optimization (PSO-SVM), were also applied in the same way as AeDE-SVM to provide a fair performance evaluation comparison. Table 4 showed the summary of optimized SVM parameters as well as the performance evaluation among three different classifiers.

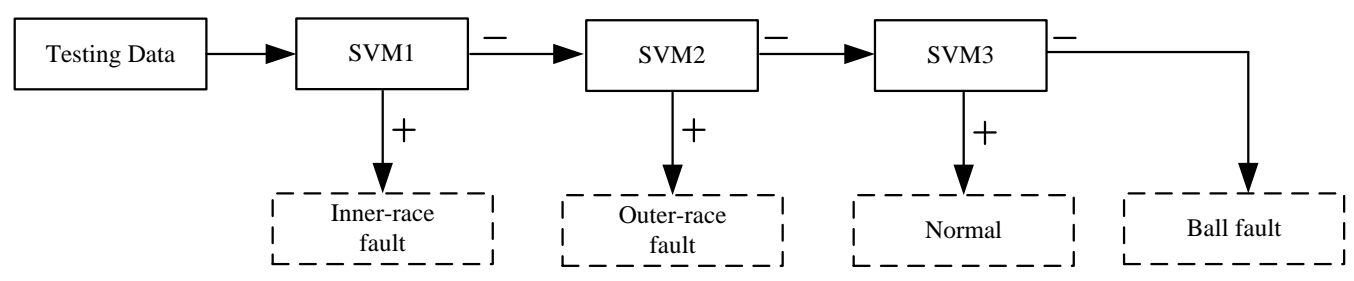

Figure 3: Multiple binary SVM classification model

Table 4: The Summary of different SVM parameter optimization techniques on roller bearing fault detection

\begin{tabular}{|rcccccc|}
\hline \multicolumn{1}{|c}{ Method } & $\begin{array}{c}\text { Training } \\
\text { samples }\end{array}$ & $\begin{array}{c}\text { Test } \\
\text { samples }\end{array}$ & Optimal C & Optimal $\sigma$ & Cost time (s) & $\begin{array}{c}\text { Average Error } \\
\text { Rate }(\%)\end{array}$ \\
\hline AeDE-SVM1 & 308 & 132 & 11715.32 & 20.64 & 35.82 & $\mathbf{0}$ \\
PSO-SVM1 & 308 & 132 & 22319.37 & 30.78 & 38.10 & 0 \\
GA-SVM1 & 308 & 132 & 20456.15 & 29.15 & 58.15 & 0 \\
\hline AeDE-SVM2 & 241 & 99 & 3309.34 & 15.34 & 29.91 & $\mathbf{0}$ \\
PSO-SVM2 & 241 & 99 & 9759.12 & 16.84 & 34.80 & 0.1010 \\
GA-SVM2 & 241 & 99 & 14545.42 & 30.67 & 62.58 & 0.1010 \\
\hline AeDE-SVM3 & 154 & 66 & 19601.37 & 17.60 & 23.96 & $\mathbf{0}$ \\
PSO-SVM3 & 154 & 66 & 5171.29 & 8.02 & 24.87 & $\mathbf{0}$ \\
GA-SVM3 & 154 & 66 & 8005.80 & 13.50 & 38.71 & $\mathbf{0}$ \\
\hline
\end{tabular}

With the same training and testing samples, AeDE-SVM perfectly achieved minimum error rate (almost $0 \%$ ) while requiring shortest processing time. The difference in average error rate is generally almost $0 \%$ in all methods, however, the processing time of the AeDE-SVMs in all classification stages were always faster than the others. In details, in the first classification stage, all optimized classifiers achieved a great average error rate of $0 \%$, AeDE-SVM1 took only 35.82 seconds to recognize 132 testing patterns. This computational time was 3 seconds faster than the PSO-SVM1 and even almost 40\% faster than GA-SVM1. The AeDE-SVM2 even performed 50\% faster than GA-SVM2 while still achieving better classification accuracy. The results were similar in the final classification stage.

Moreover, the optimal values for C and sigma for each SVM classifiers in any particular stage were not proportional to the cost time and accuracy. The optimal parameters of AeDE-SVM, which provided shortest computational time and best accuracy, were always higher than the others, while, those parameters of PSOSVM, which provided $2^{\text {nd }}$ shortest computational time, were smallest values among corresponding parameters. Therefore, PSO-SVM and GA-SVM parameter optimization algorithm were properly trapped 
in some local minima with longer optimizing time or more complex decision boundary, compared to global minima optimized by AeDE-SVM.

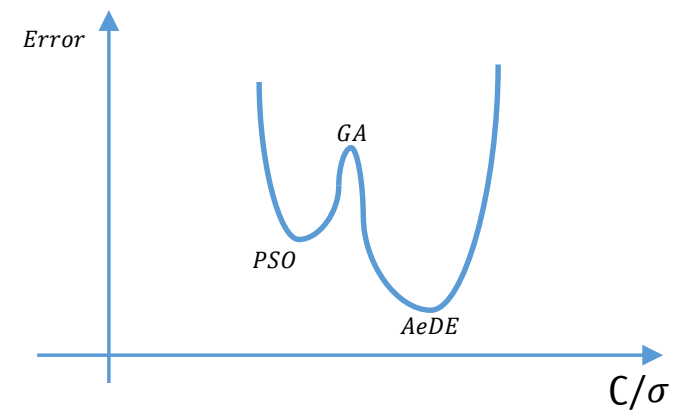

Figure 4: Demonstration of optimization problem.

Table 5: CEEMD-SVD Feature Extraction and AeDE-SVM Classification Details

\begin{tabular}{|c|c|c|c|c|c|c|c|c|}
\hline \multirow{2}{*}{$\begin{array}{l}\text { Test samples } \\
\text { (1) IR fault }\end{array}$} & \multicolumn{4}{|c|}{$\begin{array}{l}\text { Singular value of fault feature } \\
\qquad \sigma_{X, x}\end{array}$} & \multirow{2}{*}{$\begin{array}{c}\begin{array}{c}\text { AeDE- } \\
\text { SVM1 } \\
\text { classifier }\end{array} \\
(+1)\end{array}$} & \multirow[t]{2}{*}{$\begin{array}{l}\text { AeDE - } \\
\text { SVM2 } \\
\text { classifier }\end{array}$} & \multirow[t]{2}{*}{$\begin{array}{l}\text { AeDE - } \\
\text { SVM3 } \\
\text { classifier }\end{array}$} & \multirow{2}{*}{$\begin{array}{c}\begin{array}{c}\text { Identification } \\
\text { results }\end{array} \\
\text { Inner-race fault }\end{array}$} \\
\hline & 8.373 & 2.423 & 1.907 & 1.698 & & & & \\
\hline (2) IR fault & 8.025 & 2.423 & 2.039 & 1.667 & $(+1)$ & & & Inner-race fault \\
\hline (3) IR fault & 8.322 & 2.391 & 2.095 & 1.632 & $(+1)$ & & & Inner-race fault \\
\hline (4) IR fault & 8.308 & 2.467 & 2.113 & 1.654 & $(+1)$ & & & Inner-race fault \\
\hline (5) IR fault & 8.481 & 2.4 & 2.034 & 1.749 & $(+1)$ & & & Inner-race fault \\
\hline (6) IR fault & 8.152 & 2.668 & 2.038 & 1.568 & $(+1)$ & & & Inner-race fault \\
\hline (7) OR fault & 7.831 & 0.76 & 0.869 & 0.619 & $(-1)$ & $(+1)$ & & Outer-race fault \\
\hline (8) OR fault & 7.746 & 0.819 & 0.875 & 0.531 & $(-1)$ & $(+1)$ & & Outer-race fault \\
\hline (9) OR fault & 7.57 & 0.789 & 0.865 & 0.638 & $(-1)$ & $(+1)$ & & Outer-race fault \\
\hline (10) OR fault & 7.652 & 0.705 & 0.8 & 0.564 & $(-1)$ & $(+1)$ & & Outer-race fault \\
\hline (11) OR fault & 7.782 & 0.758 & 0.843 & 0.614 & $(-1)$ & $(+1)$ & & Outer-race fault \\
\hline (12) OR fault & 9.069 & 1.064 & 0.784 & 0.546 & $(-1)$ & $(+1)$ & & Outer-race fault \\
\hline (13) Normal & 4.189 & 0.234 & 0.816 & 0.504 & $(-1)$ & $(-1)$ & $(+1)$ & Normal \\
\hline (14) Normal & 3.995 & 0.238 & 0.916 & 0.538 & $(-1)$ & $(-1)$ & $(+1)$ & Normal \\
\hline (15) Normal & 4.272 & 0.221 & 0.876 & 0.546 & $(-1)$ & $(-1)$ & $(+1)$ & Normal \\
\hline (16) Normal & 3.722 & 0.238 & 0.825 & 0.536 & $(-1)$ & $(-1)$ & $(+1)$ & Normal \\
\hline (17) Normal & 4.043 & 0.241 & 0.869 & 0.527 & $(-1)$ & $(-1)$ & $(+1)$ & Normal \\
\hline (18) Normal & 4.358 & 0.242 & 0.86 & 0.507 & $(-1)$ & $(-1)$ & $(+1)$ & Normal \\
\hline (19) Ball fault & 0.631 & 0.101 & 0.987 & 0.45 & $(-1)$ & $(-1)$ & $(-1)$ & Ball fault \\
\hline (20) Ball fault & 0.633 & 0.122 & 1.055 & 0.379 & $(-1)$ & $(-1)$ & $(-1)$ & Ball fault \\
\hline (21) Ball fault & 0.610 & 0.095 & 0.959 & 0.31 & $(-1)$ & $(-1)$ & $(-1)$ & Ball fault \\
\hline (22) Ball fault & 0.636 & 0.088 & 0.898 & 0.351 & $(-1)$ & $(-1)$ & $(-1)$ & Ball fault \\
\hline (23) Ball fault & 0.619 & 0.094 & 1.039 & 0.38 & $(-1)$ & $(-1)$ & $(-1)$ & Ball fault \\
\hline (24) Ball fault & 0.619 & 0.146 & 0.895 & 0.352 & $(-1)$ & $(-1)$ & $(-1)$ & Ball fault \\
\hline
\end{tabular}

Besides, Table 5 showed the detailed results of the proposed method in which the CEEMD-SVD feature extraction process successfully transformed the time series continuous signal into four-dimensional competitive feature vectors in each class. Obviously, the new extracted values in each class were quite unique to the others, thus, significantly improved the competitiveness of the input data and generally contributed to provide the extremely low average error rate (mostly less then $0.2 \%$ ).

\section{CONCLUSION}

In this paper, a generalized method for SVM parameter optimization based on AeDE algorithm was firstly introduced. Moreover, the integration between CEEMD and SVD provided an efficient feature reduction method which transformed a long time series data into a smaller number of highly competitive feature set. The roller bearing vibrating signals were used to evaluate the proposed method. As the results, most of the classifiers achieved good results (less than $2 \%$ of classification error) due to the superior of CEEMD-SVD 
feature extraction method. By providing the negligible difference of average classification error among classifiers, AeDE-SVM showed a great and stable performance, especially processing time benefit.

\section{ACKNOWLEDGMENT}

\section{REFERENCES}

[1] Dreiseitl, S., et al., A comparison of machine learning methods for the diagnosis of pigmented skin lesions. J Biomed Inform, 2001. 34(1): p. 28-36.

[2] Matheny, M.E., et al., Effects of SVM parameter optimization on discrimination and calibration for postprocedural PCI mortality. J Biomed Inform, 2007. 40(6): p. 688-97.

[3] Glasmachers, T. and C. Igel, Gradient-based adaptation of general gaussian kernels. Neural Computation, 2005. 17(10): p. 2099-2105.

[4] Chapelle, O., et al., Choosing multiple parameters for support vector machines. Machine Learning, 2002. 46(13): p. 131-159.

[5] Chung, K.M., et al., Radius margin bounds for support vector machines with the RBF kernel. Neural Computation, 2003. 15(11): p. 2643-2681.

[6] Friedrichs, F. and C. Igel, Evolutionary tuning of multiple SVM parameters. Neurocomputing, 2005. 64: p. 107117.

[7] Ao, H., et al., Backtracking Search Optimization Algorithm and its Application to Roller Bearing Fault Diagnosis. International Journal of Acoustics and Vibration, 2016. 21(4): p. 445-452.

[8] Lim, W.H. and N.A.M. Isa, An adaptive two-layer particle swarm optimization with elitist learning strategy. Information Sciences, 2014. 273: p. 49-72.

[9] Zhang, H.H., et al., A two-stage sequential linear programming approach to IMRT dose optimization. Phys Med Biol, 2010. 55(3): p. 883-902.

[10] Pinto Mariano, A., et al., Optimization strategies based on sequential quadratic programming applied for a fermentation process for butanol production. Appl Biochem Biotechnol, 2009. 159(2): p. 366-81.

[11] Choueiki, M.H. and C.A. Mount-Campbell, Training data development with the D-optimality criterion. IEEE Trans Neural Netw, 1999. 10(1): p. 56-63.

[12] Ho-Huu, V., et al., An adaptive elitist differential evolution for optimization of truss structures with discrete design variables. Computers \& Structures, 2016. 165: p. 59-75.

[13] Storn, R. and K. Price, Differential evolution - A simple and efficient heuristic for global optimization over continuous spaces. Journal of Global Optimization, 1997. 11(4): p. 341-359.

[14] Demertzis, K. and L. Iliadis. Adaptive Elitist Differential Evolution Extreme Learning Machines on Big Data: Intelligent Recognition of Invasive Species. 2017. Cham: Springer International Publishing.

[15] Li, M.J., et al., An Improved Method Based on CEEMD for Fault Diagnosis of Rolling Bearing. Advances in Mechanical Engineering, 2014.

[16] Zhao, L.Y., W. Yu, and R.Q. Yan, Rolling Bearing Fault Diagnosis Based on CEEMD and Time Series Modeling. Mathematical Problems in Engineering, 2014.

[17] Mocellin, S., et al., Support vector machine learning model for the prediction of sentinel node status in patients with cutaneous melanoma. Ann Surg Oncol, 2006. 13(8): p. 1113-22.

[18] Yeh, J.R., J.S. Shieh, and N.E. Huang, Complementary Ensemble Empirical Mode Decomposition: A Novel Noise Enhanced Data Analysis Method. Advances in Data Science and Adaptive Analysis, 2010. 2(2): p. 135-156.

[19] Niu, M.F., et al., A novel hybrid decomposition-and-ensemble model based on CEEMD and GWO for short-term PM2.5 concentration forecasting. Atmospheric Environment, 2016. 134: p. 168-180.

[20] Chen, D.Y., J.H. Lin, and Y.P. Li, Modified complementary ensemble empirical mode decomposition and intrinsic mode functions evaluation index for high-speed train gearbox fault diagnosis. Journal of Sound and Vibration, 2018. 424: p. 192-207. 


\section{PHƯƠNG PHÁP TỐI UUU HÓA THÔNG SỐ MÁY HÕ̃ TRỢ VECTOR DỰA TRÊN THUẠTT TOÁN AEDE VÀ ỨNG DỤNG ĐỂ CHẨN ĐOÁN HƯ HỎNG Ổ LĂN}

Tóm tắt. Thông số vận hành của máy véc tơ hỗ trợ có ảnh hưởng lớn đến tỉ lệ chính xác trong quá trình phân lớp. Việc hiệu chỉnh các thông số này luôn gây khó khăn cho các nhà khoa học. Bài báo này giới thiệu một phương pháp tối ưu hóa tham số máy véc tơ hỗ trợ (SVM) dựa vào thuật toán tiến hóa vi phân thích ứng (AeDE). Sau đó, bộ phân lớp AeDE-SVM được ứng dụng để chẩn đoán hư hỏng ổ lăn bằng cách kết hợp phương pháp CEEMD và phương pháp phân rã giá trị riêng (SVD). Đầu tiên, tín hiệu gia tốc dao động được phân tích thành các Instrinsic Mode Function (IMF) bằng phương pháp CEEMD. Sau đó, các ma trận thuộc tính khởi tạo được trích xuất từ các hàm IMF bởi kỹ thuật SVD để có được các giá trị riêng. Cuối cùng, các giá trị này được sử dụng như véc tơ đầu vào của bộ phân lớp AeDE-SVM. Kết quả thực nghiệm cho thấy rằng hệ thống kết hợp giữa bộ phân lớp AeDE-SVM và hệ trích xuất đặc tính CEEMD-SVD cho tỉ lệ phân lớp chính xác cao hơn và tiêu tốn ít thời gian hơn so với các phương pháp khác.

Từ khóa. Thuật toán tiến hóa vi phân thích ứng (AeDE), phương pháp CEEMD, Hư hỏng ổ lăn, Phân rã giá trị riêng (SVD), Máy véc tơ hỗ trợ (SVM).

Ngày nhận bài: 22/11/2018

Ngày chấp nhận đăng: 17/05/2019 